\title{
Lidil
}

Revue de linguistique et de didactique des langues

$58 \mid 2018$

L'enseignement et l'apprentissage de l'écrit académique à l'aide de corpus numériques

\section{Un dictionnaire basé sur corpus pour une aide à la rédaction universitaire}

A Corpora-Based Dictionary for Academic Writing

\section{Thi Thu Hoai Tran et Achille Falaise}

\section{CpenEdition}

\section{Journals}

Édition électronique

URL : http://journals.openedition.org/lidil/5378

DOI : 10.4000/lidil.5378

ISSN : 1960-6052

Éditeur

UGA Éditions/Université Grenoble Alpes

Édition imprimée

ISBN : 978-2-37747-064-8

ISSN : $1146-6480$

Référence électronique

Thi Thu Hoai Tran et Achille Falaise, «Un dictionnaire basé sur corpus pour une aide à la rédaction universitaire », Lidil [En ligne], 58 | 2018, mis en ligne le 02 novembre 2018, consulté le 03 mai 2019. URL : http://journals.openedition.org/lidil/5378 ; DOI : 10.4000/lidil.5378

Ce document a été généré automatiquement le 3 mai 2019.

(c) Lidil 


\title{
Un dictionnaire basé sur corpus pour une aide à la rédaction universitaire
}

\author{
A Corpora-Based Dictionary for Academic Writing
}

Thi Thu Hoai Tran et Achille Falaise

\section{Introduction}

Ce travail s'inscrit dans la lignée de ceux de Tutin (2007), Chambers (2010) et Boulton et Tyne (2014), qui traitent de l'introduction de corpus dans l'enseignement/ apprentissage $\mathrm{du}$ français en tant que langue étrangère. Dans un objectif d'aide à la rédaction scientifique, nous nous intéressons au lexique transdisciplinaire scientifique (Tutin, 2010) et plus précisément aux marqueurs discursifs qui servent à structurer le texte, comme moyen pour aider les apprenants à acquérir les spécificités du discours académique. En effet, plusieurs études ont relevé des difficultés lexicales des apprenants pour la rédaction de productions écrites. Les erreurs peuvent être d'ordre syntaxique ou sémantique (Paquot, 2010 ; Osborne, 1994 ; De Cock, 2004) ou relever de la combinatoire lexicale (Simard, 1994). Les apprenants rencontrent notamment des difficultés en utilisant des séquences récurrentes (Howarth, 1998; Gledhill, 2000 ; De Cock, 2004). Cet article porte sur les expérimentations que nous avons menées auprès des étudiants non natifs afin de les familiariser avec quelques propriétés des écrits scientifiques en français, en recourant aux avantages des corpus informatisés et du TAL (Traitement automatique des langues). Nous rejoignons Wray (2002) pour affirmer qu'une connaissance des éléments phraséologiques fréquents dans ces écrits permettrait aux étudiants d'acquérir des normes stylistiques d'un genre particulier, et de mieux s'intégrer dans une communauté.

2 Dans ce travail, nous insistons sur l'importance des corpus dans l'enseignement/ apprentissage des phénomènes langagiers. Depuis les premiers travaux de Johns (1991) sur le data-driven learning, plusieurs travaux démontrent le rôle des corpus en didactique 
des langues (Chambers, 2010; Boulton \& Tyne, 2014). Les corpus permettent aux étudiants de travailler sur des données authentiques, et représentent donc pour eux un réel intérêt. Ces ressources favorisent une prise de conscience des phénomènes linguistiques (Landure, 2013), un développement de l'autonomisation et la capacité de responsabilisation.

Malgré les avantages des corpus, leur introduction en classe de langue se heurte à de nombreuses réticences comme l'ont remarqué avec justesse Cavalla et Loiseau (2013). Ce phénomène s'explique par les difficultés liées aux outils, mais aussi à l'enseignant et aux apprenants. Premièrement, force est de constater que les corpus ne proposent pas une exploitation directe des données. Deuxièmement, il manque encore des formations destinées aux enseignants de FLE (français langue étrangère) sur l'utilisation de ces outils. Nous sommes de même avis que Johns(1991) et Mauranen(2004) sur le rôle de l'enseignant pour la réussite de l'utilisation des corpus en classe de langue. L'enseignant doit avant tout prendre conscience de l'utilité de ces outils. Enfin, une autre difficulté, d'ordre cognitif, vient des apprenants. Certes, les corpus offrent un large choix d'exemples, mais la masse d'informations risque d'intimider les apprenants et de les faire tomber rapidement dans la surcharge cognitive. C'est pourquoi l'enseignant joue un rôle important dans la sélection des exemples pertinents en fonction des objectifs d'apprentissage.

4 Notre recherche se situe donc à l'intersection de plusieurs domaines, à savoir la linguistique de corpus, le TAL, et la didactique du français sur objectif universitaire (FOU) (Mangiante \& Parpette, 2011). Cet article décrit une investigation visant à trouver une mise à disposition optimale des corpus pour les apprenants et les enseignants en nous appuyant sur les remarques des utilisateurs. En effet, nous souhaitons tirer profit des avantages du TAL pour mettre en place une ressource pour une aide à la rédaction universitaire auprès d'un public allophone. Les expérimentations que nous avons menées apportent des retours enrichissants pour la conception d'un outil adaptable à un public FLE aussi bien sur le plan formel que sur le contenu.

5 Nous présentons dans le cadre de ce travail un outil qui allie les avantages de la linguistique de corpus, les résultats de l'analyse linguistique et les exploitations didactiques au service de la rédaction scientifique. C'est dans cette perspective de permettre au corpus de jouer pleinement son rôle de ressource lexicale que le projet Dicorpus $^{1}$ a débuté en 2013 (Tutin \& Falaise, 2013 ; Jacques, Hartwell \& Falaise, 2013; Falaise, 2013). Nous présenterons dans cet article la plateforme Dicorpus, son expérimentation et son évolution depuis 2013.

6 Nous allons tout d'abord évoquer quelques dictionnaires existants qui permettent de différencier notre ressource. Nous passons en revue par la suite la méthodologie de travail avant de présenter en détail notre outil Dicorpus. Enfin, nous allons décrire deux expérimentations effectuées qui guideront les futures améliorations de notre outil.

\section{Outils existants}

7 À notre connaissance, deux dictionnaires partagent le même objectif que le nôtre, c'est-àdire la mise en place d'un outil d'aide à la rédaction. Il nous semble nécessaire d'analyser l'organisation de ces deux dictionnaires avant d'expliciter le fonctionnement de notre outil. Il est à noter que les deux dictionnaires ne sont pas accessibles au grand public. 
8 Le dictionnaire ARTES $^{2}$ (fig. 1) est un outil d'aide à la rédaction et à la traduction en langues de spécialité, développé par l'équipe de recherche de l'UFR EILA et du CLILLACARP de l'université Paris Diderot. Ce dictionnaire est constitué dans une perspective contrastive. Les éléments phraséologiques (les suites de mots) sont organisés d'après leurs fonctions discursives. Par exemple, pour introduire une transition, l'utilisateur a à sa disposition quelques exemples correspondants.

Ayant la même optique fonctionnelle, le dictionnaire LEAD (Louvain English Academic Purposes Dictionary - Granger \& Paquot, 2010) se compose de 900 mots et expressions, relevés dans un grand corpus de textes académiques (dont une sélection du British National Corpus), ainsi que de corpus d'apprenants (English as a Foreign Language) dont la langue maternelle est, pour une grande majorité, le français. L'objectif principal du dictionnaire est de mettre à disposition des utilisateurs des moyens linguistiques de base. En effet, il offre aux utilisateurs une riche description du lexique transdisciplinaire des écrits scientifiques en anglais et porte essentiellement sur la phraséologie, en particulier les collocations et les segments répétés.

Figure 1. - Dictionnaire ARTES.

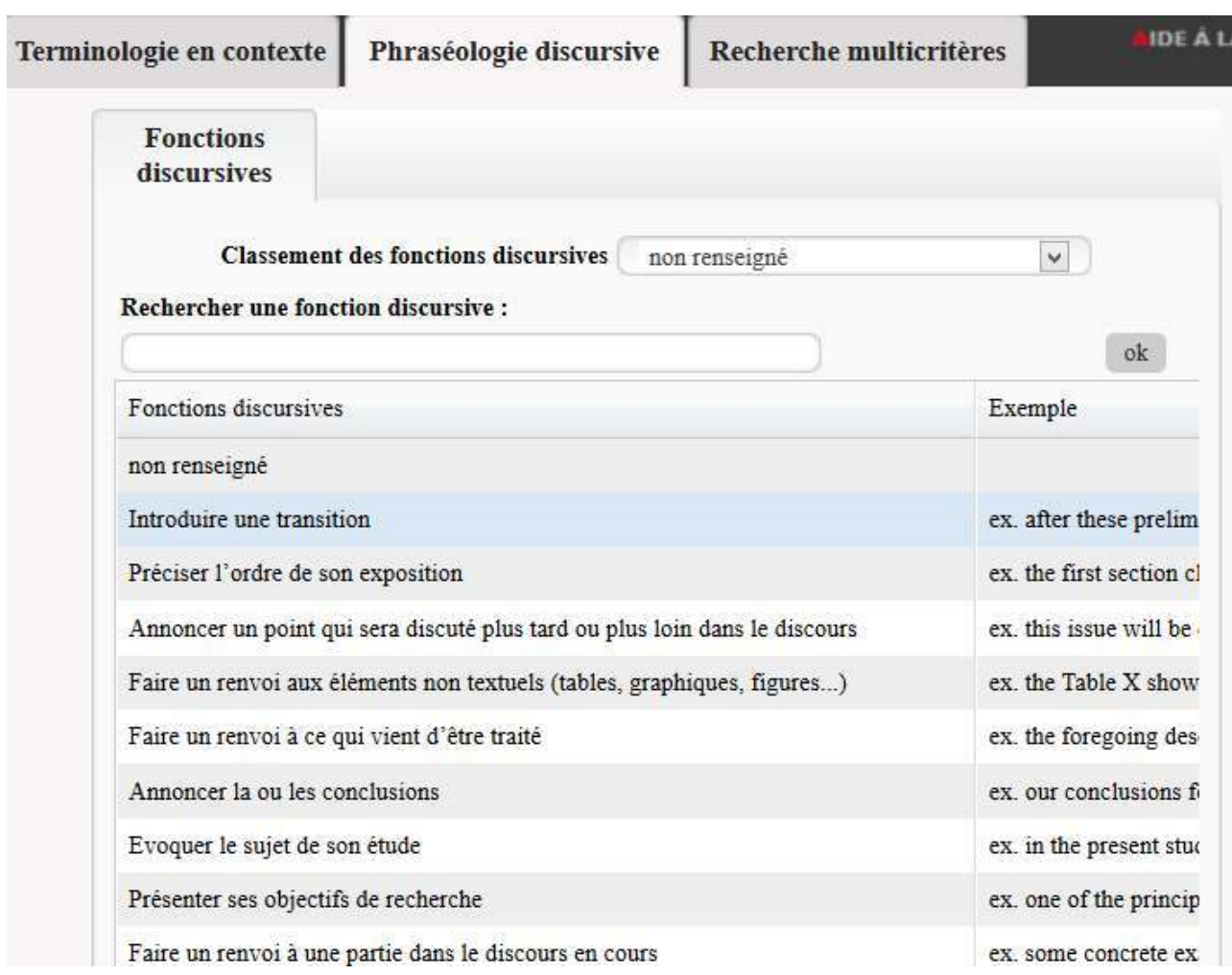

Dans le LEAD (fig. 2), les utilisateurs peuvent accéder au dictionnaire par différents modes comme le mode sémasiologique (de la forme au sens) qui permet d'effectuer une recherche par le lemme et le mode onomasiologique (du sens à la forme) par le biais d'une liste des fonctions rhétoriques ou organisationnelles du discours scientifique (au total 18 fonctions). Pour chaque fonction, l'utilisateur peut accéder à une liste des éléments lexicaux typiques dans le discours scientifique et pour chaque catégorie grammaticale (noms, verbes, adjectifs, adverbes, conjonctions et prépositions).

11 En outre, l'utilisateur a également la possibilité de choisir sa langue maternelle et de faire des recherches par des entrées lexicales traduites dans cette langue. Le choix de la langue 
maternelle des apprenants joue un rôle important dans l'identification des erreurs et des problèmes spécifiques de chaque langue. Il sert aussi à analyser les influences sur la traduction de la langue maternelle à la langue cible. Toutes les erreurs commises par les apprenants sont enregistrées et signalées par la suite aux apprenants si ceux-ci choisissent la même langue maternelle.

Figure 2. - Accès onomasiologique dans LEAD (d'après Granger \& Paquot, 2010, p. 323).

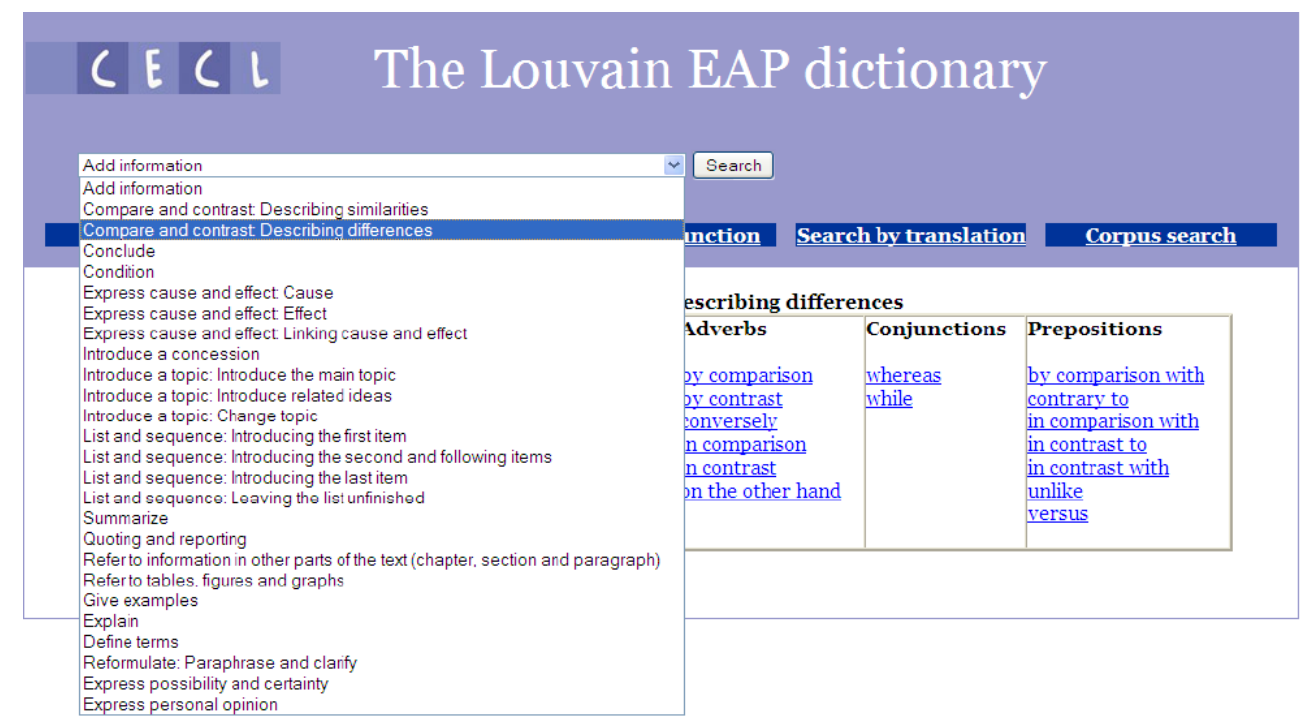

\section{Méthodologie}

Dans le projet Dicorpus, nous nous appuyons sur quatre ressources, qui seront détaillées dans cette partie :

- un corpus, dans lequel l'enseignant sélectionne des exemples ;

- la base d'exemples ainsi constituée par l'enseignant ;

- une base de métadonnées lexicographiques concernant ces exemples, créée par l'enseignant ;

- et enfin l'interface utilisateur Dicorpus, qui permet la consultation, par les apprenants, des deux bases précédemment citées (exemples et métadonnées associées).

Nous avons mené deux expérimentations auprès d'étudiants non natifs pendant deux années consécutives, en 2015-2016 et 2016-2017, dans l'objectif de comprendre comment ceux-ci utilisent le Dicorpus, et ainsi de pouvoir proposer par la suite un outil qui répond au mieux à leurs attentes. La préparation du dictionnaire pour la classe a été prise en charge par une seule enseignante. Chaque groupe-classe comprend une vingtaine d'étudiants. Ceux-ci ont passé trois séances à travailler sur Dicorpus en classe, après quoi ils ont été invités à travailler chez eux sur cet outil. Il est à noter que les étudiants doivent rendre un devoir qui correspond à une partie textuelle dans un écrit universitaire, par exemple l'introduction ou la conclusion. 


\subsection{Le corpus Scientext}

Le corpus Scientext (Tutin \& Grossmann, 2012) est un méta-corpus arboré, annoté avec l'analyseur Syntex (Bourigault, 2007), et constitué de plusieurs corpus de textes scientifiques. L'un d'entre eux est particulièrement utile pour le projet Dicorpus ; il s'agit du corpus de textes scientifiques en français ( 205 documents, 5 millions de mots). Ce corpus a été constitué dans le cadre du projet Scientext ${ }^{3}$, afin d'étudier les marques transdisciplinaires du positionnement et du raisonnement dans les écrits scientifiques.

Par exemple, on peut voir que la locution « au passage » à valeur rhétorique est beaucoup moins utilisée par les apprenants que par les scientifiques (fig. 3), en comparant le corpus Scientext avec un corpus de mémoires de master de SHS, rédigés par des apprenants du français (corpus de 39 mémoires, 600000 mots, collecté au LIDILEM).

Figure 3. - Fréquence relative de " au passage » à valeur rhétorique dans le corpus d'apprenants du français et dans les textes de SHS du corpus Scientext (161 documents, 3,9 millions de mots).

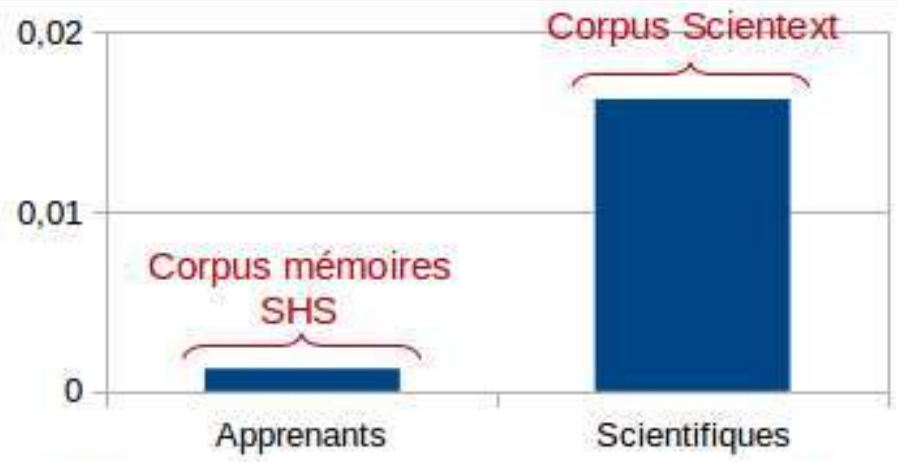

Fréquence relative de « au passage » rhètorique

D'autre part, la phraséologie du discours académique n'est pas homogène, mais varie en fonction de la disciplines, du sous-genre, ou encore des parties textuelles), et le corpus Scientext prend en compte ces différences.

Ainsi, ce corpus couvre huit disciplines (biologie, électronique, linguistique, mécanique, médecine, psychologie, sciences de l'éducation et traitement automatique des langues). Par exemple, la locution « au passage » à valeur rhétorique n'est, d'après notre corpus, pratiquement jamais utilisée en psychologie, mais est attestée en TAL, en linguistique, et particulièrement en sciences de l'éducation (fig. 4). 
Figure 4. - Fréquence relative de la locution « au passage » à valeur rhétorique dans le corpus d'apprenants du français (à gauche), et dans quatre disciplines du corpus de textes scientifiques Scientext.

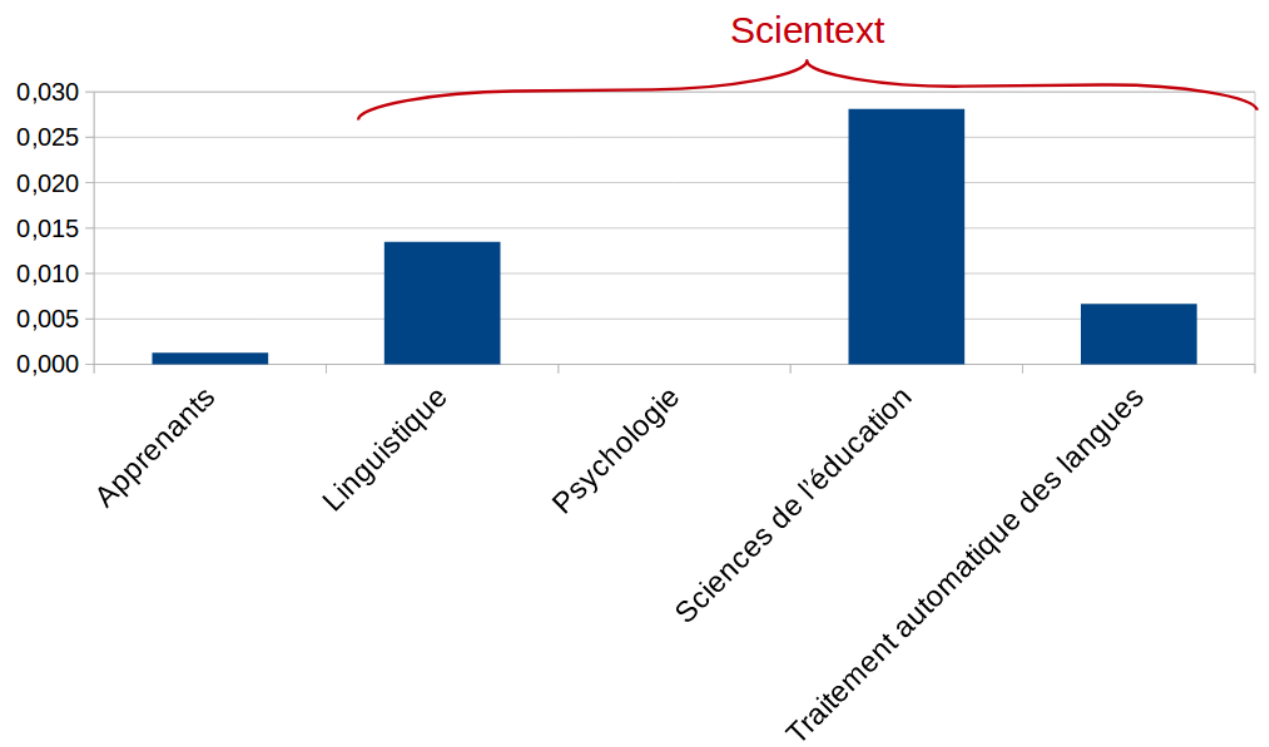

Figure 5a. - Fréquence relative de la locution « au passage » à valeur rhétorique dans un corpus d'apprenants (à gauche), et dans trois parties textuelles du corpus de textes scientifiques Scientext.

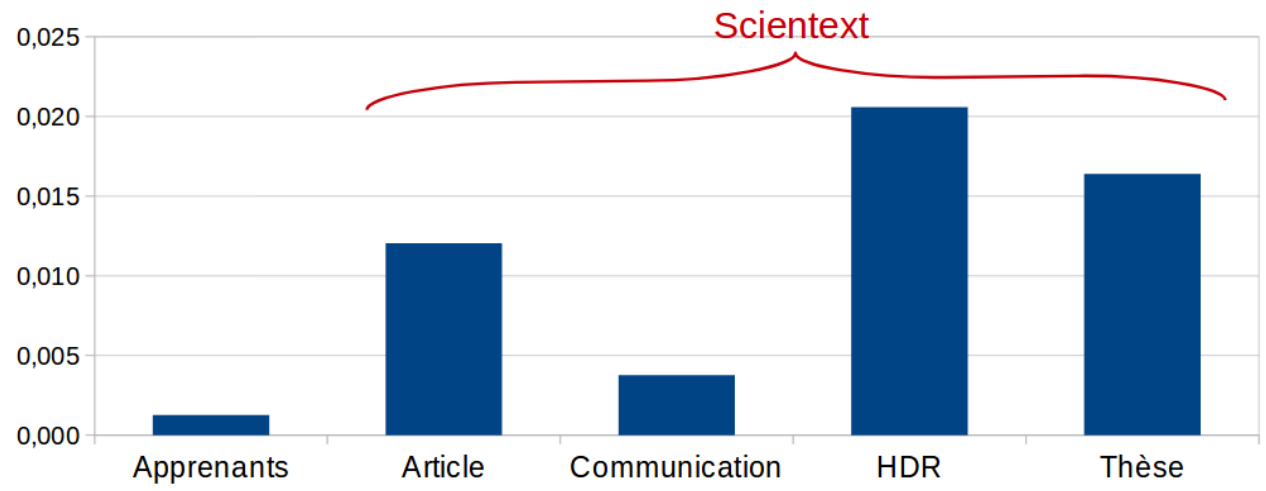

19 On observe aussi des différences importantes en fonction du sous-genre textuel (fig. 5a).

20 La structuration du corpus en parties textuelles (introduction, développement, notes, conclusion, etc.), permet de cibler les exemples en fonction de ce critère. Toujours avec notre exemple de "au passage", on constate que cette locution est beaucoup plus présente dans les notes que dans le reste du texte (fig. 5b). 
Figure 5b. - Fréquence relative de la locution " au passage » à valeur rhétorique dans un corpus d'apprenants (à gauche), et dans trois parties textuelles du corpus de textes scientifiques Scientext.

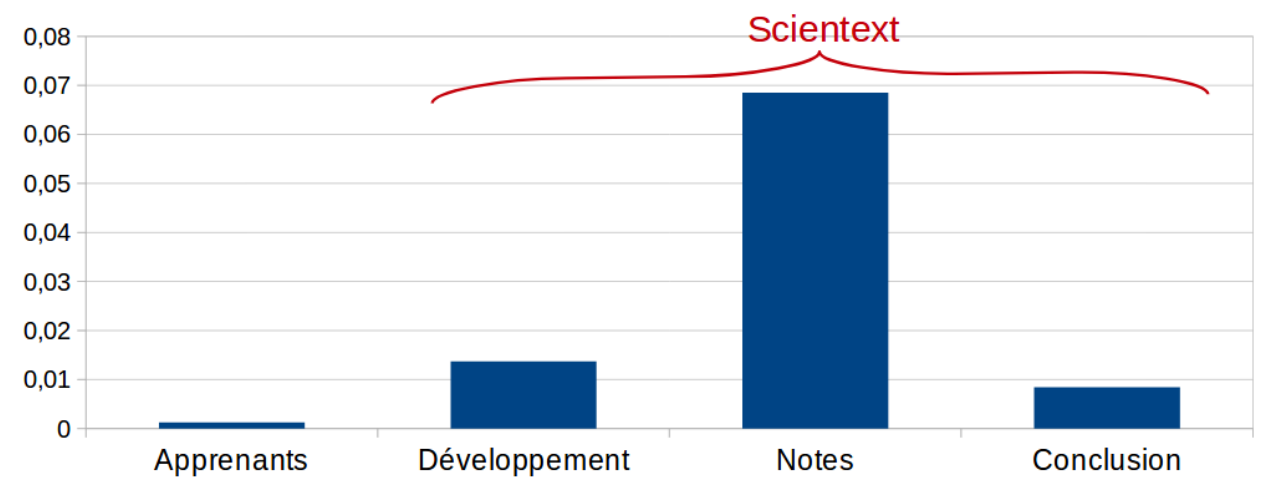

21 Le corpus Scientext a ainsi été utilisé dans plusieurs travaux portant sur l'aide à la rédaction scientifique et à la didactique de l'écrit académique: soit seul, indépendamment d'une plateforme (Hartwell \& Jacques, 2012 ; Cavalla \& Loiseau, 2013), ou bien en lien avec le projet Dicorpus (Tutin \& Falaise, 2013 ; Jacques, Hartwell \& Falaise, 2013 ; Falaise, 2013).

\subsection{La base d'exemples}

La plateforme d'exploration linguistique ScienQuest (Falaise, Tutin \& Kraif, 2011) a été initialement développée dans le cadre du projet Scientext. Elle permet d'afficher des concordances sur corpus, à l'aide d'une interface qui permet d'éviter, dans la plupart des cas, l'utilisation d'un langage de requêtes, et son ergonomie a été travaillée pour être accessible à des linguistes sans formation préalable.

Dans le cadre du projet Dicorpus, nous avons réalisé une interface spécifique pour les apprenants (infra «l'interface utilisateur Dicorpus»), mais nous n'avons pas encore effectué ce travail pour les enseignants, qui doivent utiliser l'interface classique de ScienQuest pour constituer la base d'exemples.

Pour constituer cette base, l'enseignant effectue donc une recherche dans ScienQuest (étapes 1 et 2 de la fig. 6), puis filtre les résultats qui lui semblent pertinents pour les apprenants (étape 3). Enfin, l'enseignant sauvegarde cette sélection dans un fichier qu'il télécharge (étape 4). L'ensemble de ces fichiers constitue la base d'exemples. 
Figure 6. - Étapes de la constitution d'une base d'exemples dans ScienQuest.

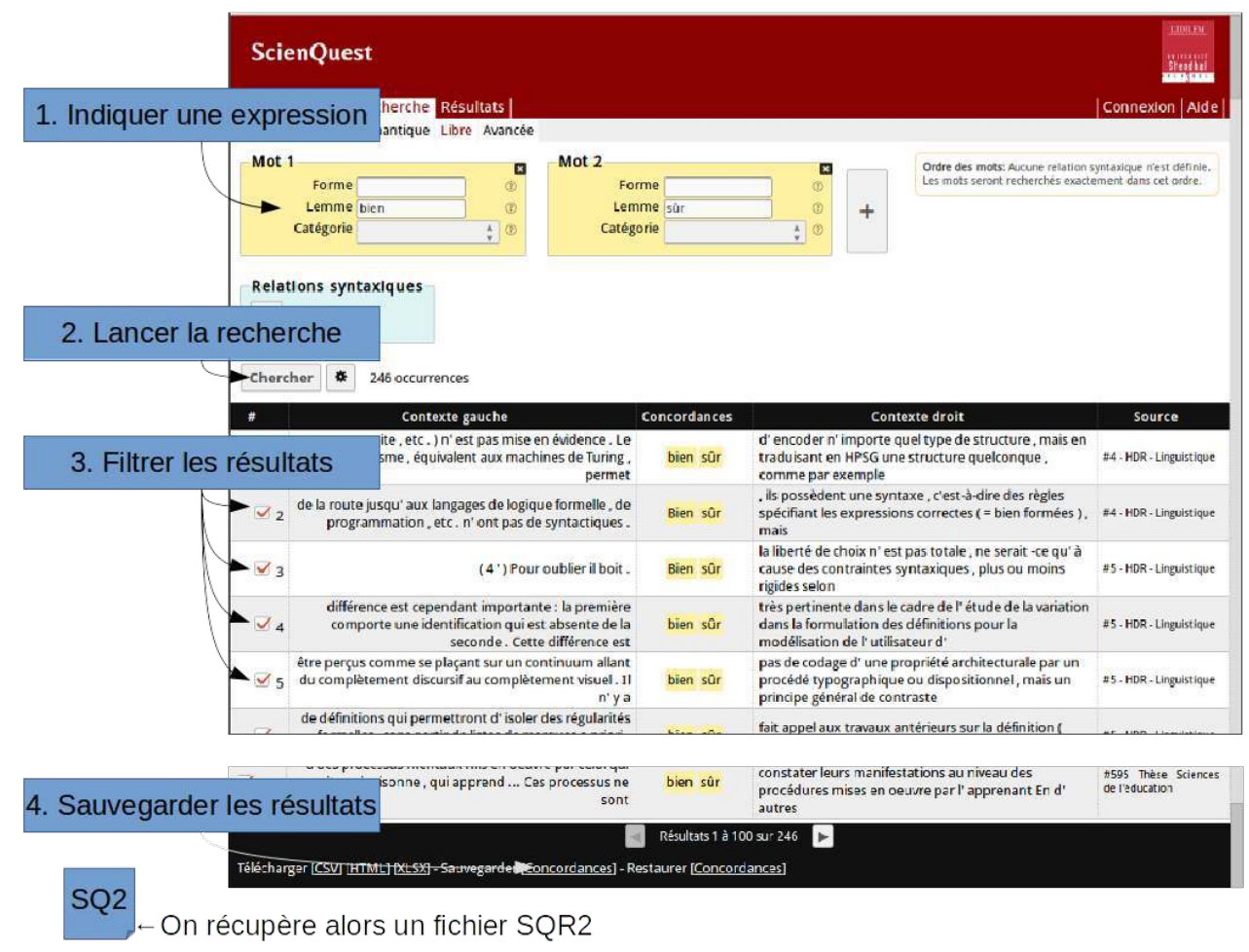

\subsection{La base lexicographique}

Afin d'organiser la base d'exemples, l'enseignant doit créer un autre fichier, qui contient les métadonnées de chaque entrée lexicale, telles que la catégorie onomasiologique (fig. 8), ainsi que les données de la notice linguistique (fig. 10 : catégorie, position dans la phrase, portée, définition, fréquence, etc.).

Techniquement, cette base consiste en un unique fichier au format CSV, que l'enseignant peut éditer avec un tableur. Chaque ligne correspond à une entrée lexicale (par exemple la locution adverbiale au passage), et chaque colonne à une métadonnée (catégorie onomasiologique, catégorie grammaticale, position dans la phrase, etc.).

\subsection{L'interface utilisateur Dicorpus}

L'interface ScienQuest, conçue pour des linguistes, n'est pas adaptée à des apprenants de langue. C'est pourquoi nous avons commencé à travailler en 2013 sur une interface « Dicorpus ", adaptée aux apprenants (Tutin \& Falaise, 2013 ; Jacques, Hartwell \& Falaise, 2013 ; Falaise, 2013), distincte de ScienQuest. Nous rappelons dans cette partie les grands principes de cette interface, et dans la partie suivante, nous présenterons les expérimentations que nous avons effectuées, ainsi que les changements fonctionnels que ces dernières nous ont conduit à opérer depuis 2013.

L'interface Dicorpus est organisée en deux grandes parties (fig. 7) :

1. L'interface lexicographique. Il s'agit d'une interface dynamique, qui prend en paramètre une base lexicographique créée par l'enseignant (cf. section précédente). Elle comprend une partie «Accès» qui permet à l'apprenant de naviguer dans le lexique jusqu'à l'entrée 
recherchée, grâce à des listes en cascade. Cet accès peut s'effectuer soit de manière onomasiologique ("accès par sens", fig. 8), soit de manière sémasiologique ("accès par expression », fig. 9). Dans tous les cas, la fréquence de chaque expression est indiquée dans les menus. La notice linguistique (fig. 10) est affichée à côté du composant d'accès.

2. L'interface corporale (exemples). Dans la première version de Dicorpus, développée en 2013, il s'agissait de la visualisation standard de ScienQuest, en concordances (comme dans fig. 6, «filtrer les résultats»). Cette visualisation a évolué dans les versions plus récentes de Dicorpus, comme nous le décrivons dans la partie suivante.

Figure 7. - Structure de l'interface de Dicorpus.
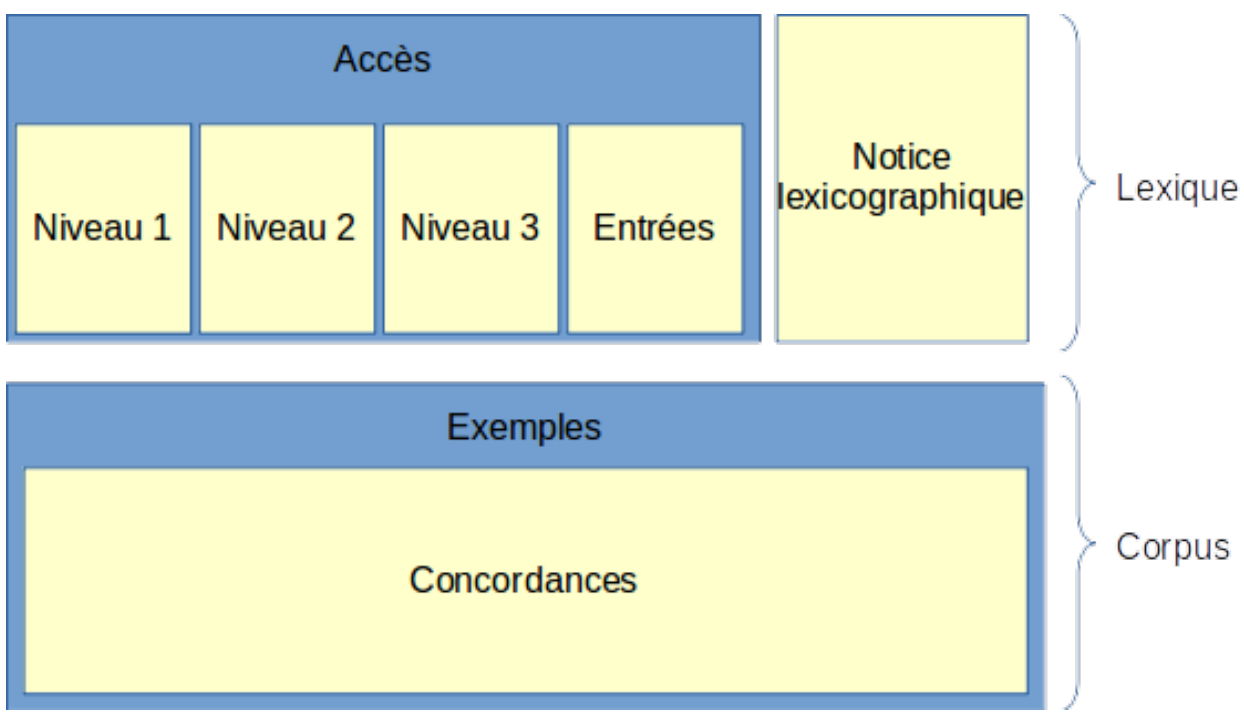

Figure 8. - Capture d'écran de l'interface de Dicorpus, composant d'accès onomasiologique (dit " accès par sens » dans l'interface).
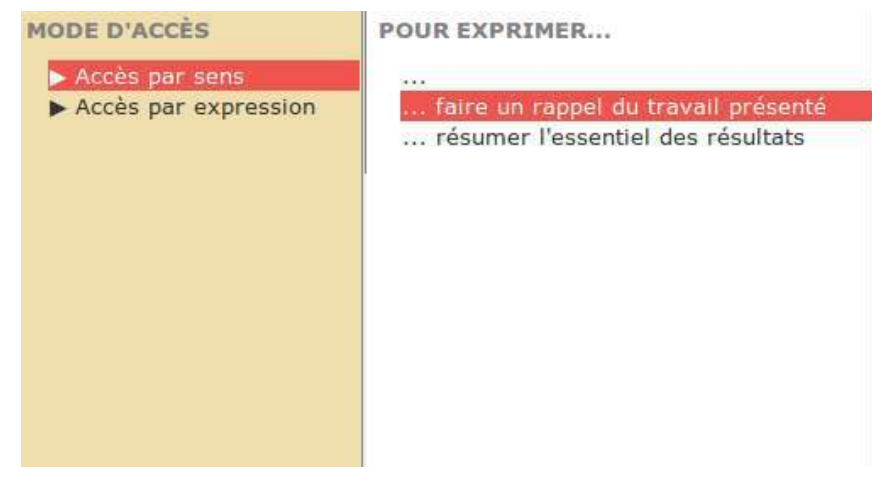

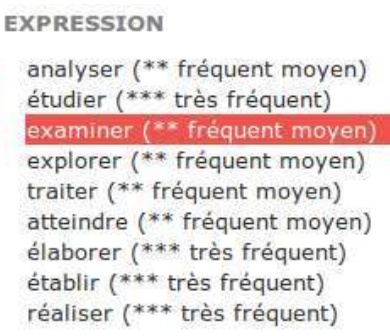


Figure 9. - Capture d'écran de l'interface de Dicorpus, composant d'accès sémasiologique (dit « accès par expression » dans l'interface).

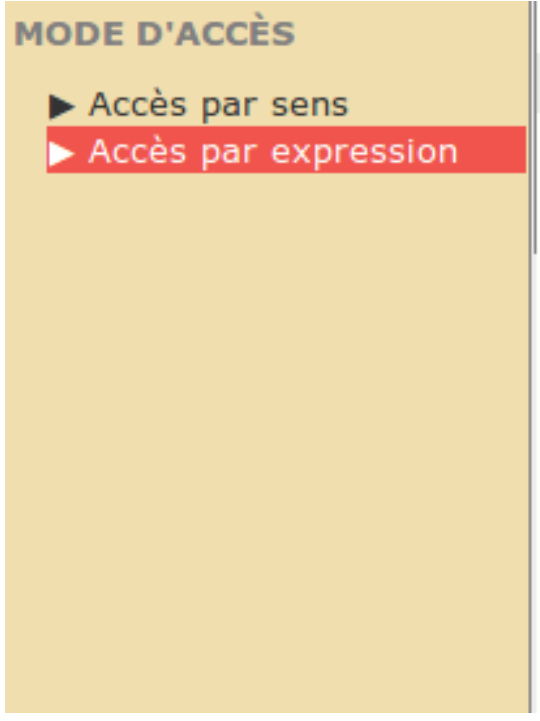

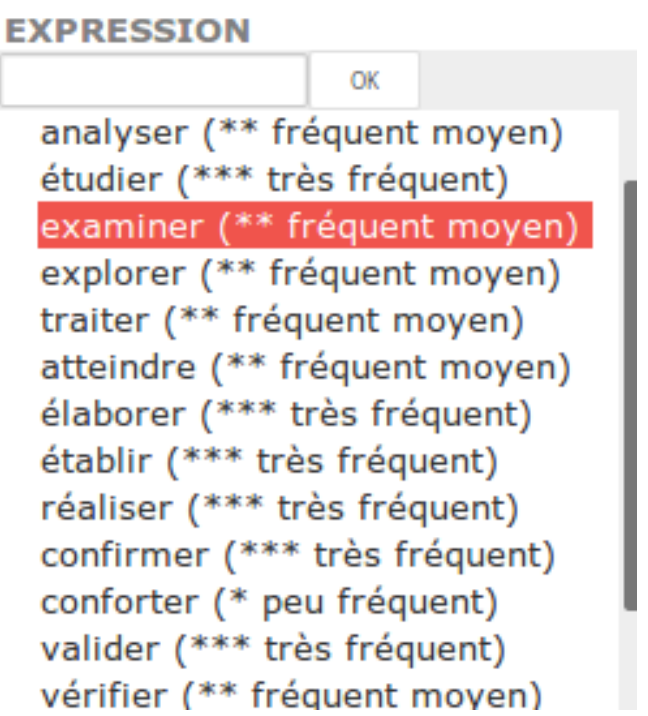

Figure 10. - Notice linguistique.

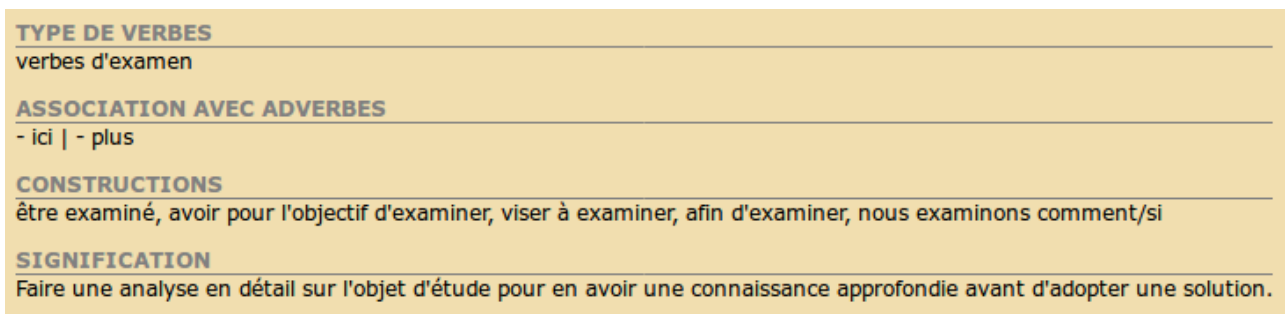

\section{Expérimentations}

Une première expérimentation sur la didactique de l'anglais ${ }^{4}$ a permis de valider l'intérêt pédagogique de Dicorpus pour croiser des données lexicales et corporales (Frérot, Rossi \& Falaise, 2014). Nous détaillerons ici deux expérimentations qui ont été effectuées auprès d'étudiants locuteurs non natifs du français, dans le cadre de la formation du DU FLEPES ${ }^{5}$, à l'université d'Artois en 2015-2016 et 2016-2017. Ces étudiants disposent d'un bagage linguistique de niveau faible à moyen (A2 à B1) et viennent de différents systèmes éducatifs (chinois, vietnamien, libyen, indien, soudanais, qatari). Cette année de préparation, considérée comme charnière, permet à ces étudiants d'acquérir un niveau de langue nécessaire pour poursuivre leurs études l'année suivante en licence ou en master dans des universités françaises. Comme ils viennent de différentes disciplines (FLE, génie civil, génie électricité, LEA, biologie, économie, finance, informatique, etc.), les modules mettent l'accent sur une préparation méthodologique, par exemple la rédaction d'un résumé, une dissertation, etc. Notre module porte essentiellement sur les écrits universitaires. Plus précisément, l'objectif de ce module est d'aider les étudiants à prendre en compte les normes spécifiques «à la française » des rapports de stage ou des mémoires, qu'ils devront rédiger plus tard dans leur cursus. 

préparatoire, et fait suite à des modules sur le résumé, le compte rendu, la synthèse et la dissertation. Nous avons mis l'accent sur un accès onomasiologique et une entrée lexicale. Notons que le module est organisé en fonction des spécificités dans des écrits universitaires, par exemple: formuler la problématique ou l'hypothèse, introduire une illustration ou un constat, reformuler une idée, etc. En particulier, nous considérons que les adverbes, principalement les marqueurs discursifs, appelés aussi les connecteurs logiques, sont des éléments qui facilitent un accès à ces écrits, comme les marqueurs qui servent à organiser le texte (dans un premier temps, tout d'abord, etc.) ou les marqueurs d'illustration (par exemple, pour illustrer, etc.).

31

cadre de nos expérimentations, nous cherchons à comprendre, dans un premier temps, les manières dont les étudiants utilisent les dictionnaires et leurs stratégies d'apprentissage. Dans un deuxième temps, nous souhaitons évaluer la perception des étudiants vis-à-vis de Dicorpus pour mieux répondre aux besoins de ce public. Dans le cadre de ces expérimentations, nous nous sommes limités à l'enseignement et l'apprentissage des marqueurs discursifs (désormais MD), les éléments qui servent à organiser un texte et à structurer un discours. La typologie des MD polylexicaux les plus fréquents dans les écrits scientifiques et l'analyse linguistique ont été faites dans une étude antérieure (Tran, 2014). Dicorpus a été introduit dans le cours comme un moyen pour enseigner les MD, et pour familiariser davantage les étudiants à un nouveau genre, l'écrit universitaire.

32 À l'issue de ces expérimentations, un questionnaire (annexe 2) a été remis aux étudiants. Les questions portent sur le comportement des étudiants lors de l'utilisation du dictionnaire et sur la représentation de Dicorpus. Il est à noter qu'en 2016-2017, un questionnaire a été distribué en début d'année universitaire avant les formations pour comprendre les habitudes ainsi que les besoins des étudiants (annexe 1). Nous avons rassemblé les résultats récoltés de deux années consécutives; les réponses ont été analysées quantitativement et qualitativement.

Concernant la forme de Dicorpus, $75 \%$ des étudiants l'apprécient par son interface conviviale et par son utilité, tandis que $25 \%$ la jugent trop simple. Quant à la recherche des informations sur le dictionnaire, la plupart des étudiants trouvent des informations nécessaires dans le dictionnaire, par exemple les synonymes, la définition de chaque expression ou les exemples correspondants. Un étudiant souligne ainsi : " Des exemples sont excellents et faciles à comprendre. Il n'est pas difficile de trouver des synonymes des mots $^{6}$.» (E) Seuls $7 \%$ des étudiants ne trouvent pas les informations nécessaires dans le dictionnaire.

Quant au contenu du dictionnaire, le premier élément qui nous intéresse est lié à la recherche d'informations. À propos de la recherche d'exemples, à notre surprise, peu d'étudiants (13\%) trouvent facilement les exemples qui se trouvent en bas de page (nécessitant de faire défiler la page vers le bas), les autres les ignorent en raison de leur position. En outre, $40 \%$ des étudiants apprécient la quantité d'exemples, mais les $60 \%$ restants sont perturbés par leur nombre important et ne savent pas quel exemple choisir. Un autre point souligné par les étudiants est en lien avec la complexité du métalangage du dictionnaire, en particulier la notion de = «portée », d'« enchainement thématique » ou bien de "séquences lexicalisées à fonction discursive». Pour la recherche des expressions ou adverbes, certains étudiants suggèrent d'ajouter une fonctionnalité pour 
chercher par des mots-clés, de les classer par ordre alphabétique ou de différencier les catégories par des couleurs différentes.

Pour résumer, les étudiants apprécient ce genre de dictionnaire et le trouvent différent par rapport aux autres dictionnaires numériques. Si l'outil est jugé utile et convivial, l'ergonomie parait peu claire. Notamment, les étudiants souhaitent avoir une vue d'ensemble des mots présents dans le dictionnaire. $56 \%$ préfèrent que les exemples soient filtrés par discipline de spécialité. «Je pense qu'il vaut mieux de choisir quelques exemples typiques pour faciliter la consultation et la compréhension. Si les étudiants veulent en regarder davantage, ils peuvent cliquer sur le plus. » (E)

L'évolution de Dicorpus concerne à la fois l'aspect technique et la conception de la base de données, en particulier dans le choix d'un métalangage plus simplifié. Dans la partie suivante, nous proposons des évolutions de l'outil basées sur ces retours.

\section{5. Évolution de Dicorpus}

À l'issue de ces expérimentations, nous avons retenu quatre problèmes principaux avec l'interface de Dicorpus, dans sa version initiale de 2013, pour les apprenants :

- l'affichage en concordances (fig. 6, "Filtrer les résultats») est perturbante pour des utilisateurs non linguistes ;

- l'affichage en colonnes des accès sémasiologiques et onmasiologiques (fig. 8 et 9) ne donnent pas une vue d'ensemble aux apprenants ;

- les liens entre les expressions ne sont pas visibles dans l'interface ;

- le classement des exemples est arbitraire (il s'agit de l'ordre d'apparition dans le corpus).

Nous proposons des solutions à ces problèmes, dont certaines sont déjà implémentées. Il s'agit des améliorations liées à l'affichage des expressions et la navigation entre les expressions, et aussi à la consultation des exemples.

\subsection{Visualisation des exemples}

39 La vue en concordances, adaptée pour des linguistes, est très inhabituelle pour des apprenants en langue. L'affichage KWIC (Key Word In Context) est déroutant et souvent trop court pour les apprenants, et par ailleurs, la référence exacte de chaque exemple n'est pas détaillée : seul est affiché un code abstrait identifiant le texte. C'est pourquoi, à l'issue de la deuxième expérimentation (2015-2016), nous avons décidé de mettre en place une vue simplifiée pour les apprenants (fig. 11), avec un affichage plus traditionnel des exemples.

\subsection{Vue d'ensemble du lexique}

Sur le plan lexical (composants d'accès onomasiologique et sémasiologique), le principal problème que nous retenons porte sur l'affichage en colonnes (fig. 3 et 4), qui n'offre pas de vue d'ensemble des expressions.

41 En mode d'accès onomasiologique en particulier, la liste des expressions n'est jamais entièrement visible, puisqu'elles sont réparties dans différents sous-menus. De plus, la vue en listes ne met pas en avant les relations entre les groupes onomasiologiques; la navigation entre les expressions est donc un peu compliquée pour les apprenants. 

classer par ordre alphabétique, par ordre de fréquence, ou bien, dans le cas de l'accès sémasiologique, par groupe onomasiologique, c'est-à-dire en regroupant les expressions partageant la même fonction rhétorique ? On peut décider de laisser l'utilisateur choisir son critère de tri, mais cela laisse entière la question du choix qui sera présenté par défaut à l'utilisateur, d'autant plus important que ce dernier ne pensera parfois pas à le modifier.

À l'heure actuelle, nous n'avons pas de réponse concrète à ces questions d'accessibilité des entrées lexicales. Toutefois, les exemples nous offrent une opportunité de mettre en place un lien entre les expressions, l'évolution qui sera détaillée dans le point suivant.

Figure 11. - Vue simplifiée des exemples pour les apprenants.

Les perspectives de recherche qu'ouvrent nos investigations nous paraissent s'inscrire dans la continuité de ce qui a été déjà exploré. Nous mesurons à la fois ce qui a été fait réussir à adapter le modèle de la TSD à des contextes nouveaux, à construire des situations et à dégager quelques principes pour cette construction et les nombreuses questions qui restent sans réponse.

[Sciences de l'éducation - HDR - Conclusion] QUELQUES APPORTS DE LA THEORIE DES SITUATIONS A LA DIDACTIQUE DES MATHEMATIQUES DANS L'ENSEIGNEMENT SECONDAIRE ET SUPERIEUR, Isabelle BLOCH

Plusieurs pistes ont commencé à être explorées pour faciliter l'exploitation de la malléabilité. La méthode et l'atelier d'ingénierie Bricoles (Caron et al., 2005) permettent aux utilisateurs de se construire une représentation compréhensible des possibilités de l'artefact. Ils permettent aux utilisateurs d'exprimer dans leur propre langage le dispositif pédagique qu'il veulent mettre en place et les aident à l'opérationnaliser par l'instrumentation ou/et l'instrumentalisation souhaitées de l'artefact. (Bouguin 2000), quant à lui, exploite la réflexivité de DARE et CoolDA l'instrumentalisation souhaitees de l'artefact. (Bouguin 2000), quant à lui, exploite la réflexivite de DARE et CoolDA
pour intégrer aux systèmes informatiques interactifs le support à l'activité coopérative de leur propre co-évolution, les transformant ainsi en systèmes expansifs.

[Sciences de l'éducation - Communication - Conclusion] Co-construire un environnement numérique adapté à un dispositif de formation (Actes de AREF), Frédéric Hoogstoel

\subsection{Navigation entre expressions}

Il n'y a actuellement pas de lien entre les expressions dans l'interface, alors que, notamment dans le cas des connecteurs discursifs, celles-ci fonctionnent souvent de manière coordonnée.

Pour remédier à ce problème, au moins dans les exemples, nous proposons de mettre en valeur, sous forme de liens hypertextes, dans chaque exemple, toutes les expressions présentes dans la base, et non plus la seule expression recherchée par l'apprenant. L'apprenant disposera ainsi d'un troisième mode d'accès aux expressions, passant directement par les exemples. Cette fonctionnalité sera implémentée dans la prochaine version de Dicorpus.

\subsection{Classement des exemples}

Dans la première version de Dicorpus, les exemples étaient classés par ordre d'apparition dans le corpus. Toutefois, nous avons identifié deux types de classements qui peuvent s'avérer utiles d'un point de vue pédagogique :

1. Un classement en fonction de critères fournis par l'apprenant, comme la discipline ou la partie textuelle qu'il est en train de rédiger;

2. Un classement par l'enseignant, afin de mettre en avant les exemples les plus pertinents - actuellement, l'enseignant peut seulement retirer des exemples, mais ne peut pas classer les exemples qu'il conserve. 
Le premier type de classement (par l'apprenant) a pu être implémenté à l'issue de la troisième expérimentation (2016-2017). Àl'avenir, nous souhaitons proposer un classement par l'enseignant.

La solution retenue pour l'instant consiste à mettre en place un panneau de configuration dans Dicorpus, intégré à partir de ScienQuest, qui permet ainsi de choisir un ou plusieurs disciplines, type de document, ou partie textuelle (fig. 12).

Figure 12. - Composant de sélection du type de texte et de la partie textuelle de ScienQuest, intégré dynamiquement dans Dicorpus.

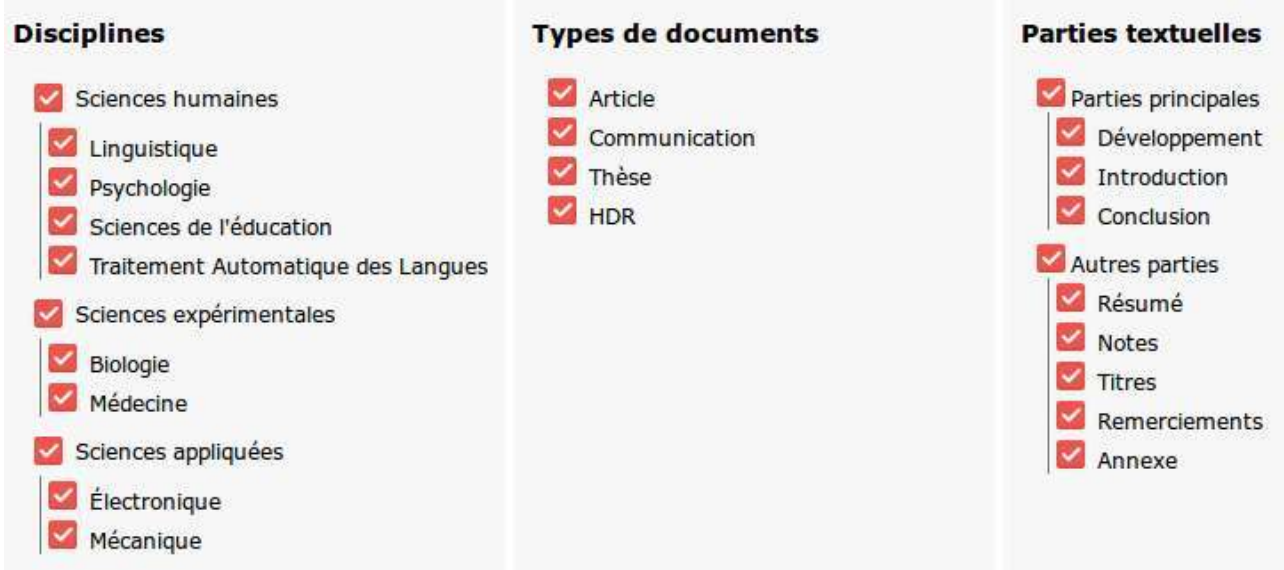

Lorsque l'apprenant a configuré les exemples, c'est-à-dire qu'il a retiré les disciplines, types de documents, ou parties textuelles qui ne l'intéressent pas (ou moins), les exemples sont triés en fonction de ces critères : ceux qui satisfont tous les critères sont affichés en premier, puis ceux qui les satisfont tous sauf un, etc. Ainsi, même si la base ne contient pas d'exemple correspondant exactement à la configuration effectuée par l'apprenant, des exemples seront néanmoins disponibles.

Quant au contenu de notre base de données, suite aux remarques des étudiants, nous avons opté pour un métalangage plus simplifié qui concerne à la fois les paramètres dont il faut tenir compte et leur explication. Les notions qui sont jugées compliquées pour les étudiants sont enlevées, par exemple la "portée » des marqueurs discursifs, bien qu'il s'agisse d'un élément important pour l'analyse linguistique, qui permet de définir la zone d'influence de chaque connecteur. En consultant chaque connecteur logique, l'utilisateur a à sa disposition des informations concernant sa définition, sa fréquence dans les écrits scientifiques, sa position dans l'énoncé et ses éventuels synonymes.

\section{Conclusion}

51 Ce travail tente de démontrer le lien entre l'analyse linguistique et le TAL dans l'enseignement/apprentissage du lexique. Le dictionnaire Dicorpus représente pour nous un moyen pour introduire les corpus en classe de langue, aussi bien auprès des étudiants que des enseignants. Pour l'enseignant, il peut y trouver des exemples pour la conception de ses cours. Pour les apprenants, le dictionnaire peut être utilisé en totale autonomie. Les expérimentations effectuées nous permettent d'évaluer comment nous pouvons adapter le dictionnaire pour mieux répondre aux besoins des étudiants. Dans l'ensemble, les étudiants semblent apprécier Dicorpus par l'interface et par le contenu qu'ils peuvent 
y trouver. Nous avons pris en compte leurs retours et leurs suggestions pour alimenter quelques fonctionnalités de l'outil. Une autre question qui émerge suite à ces expérimentations est liée à l'évaluation de leur production écrite, et à l'évaluation de l'apport de Dicorpus. Nous cherchons à comprendre en quoi Dicorpus peut aider les étudiants dans la rédaction scientifique. Une autre expérimentation est à envisager dans cette perspective où nous allons enregistrer les écrans d'ordinateur des étudiants quand ils travaillent sur Dicorpus, ce qui permettra de vérifier comment ceux-ci utilisent l'outil quand ils rédigent.

\section{BIBLIOGRAPHIE}

Boulton, Alex \& Tyne, Henry (dir.). (2014). Des documents authentiques aux corpus. Démarches pour l'apprentissage des langues. Paris : Didier.

BOURIGAULT, Didier. (2007). Un analyseur syntaxique opérationnel: SYNTEX (Mémoire d'habilitation à diriger des recherches). Université de Toulouse-Le Mirail.

Cavalla, Cristelle \& Loiseau, Mathieu. (2013). Scientext comme corpus pour l'enseignement. Dans A. Tutin \& F. Grossmann (dir.), L'écrit scientifique : du lexique au discours. Autour de Scientext (p. 163-180). Rennes : Presses universitaires de Rennes.

CHAMBERS, Angela. (2010). L'apprentissage de l'écriture en langue seconde à l'aide d'un corpus spécialisé. Revue française de linguistique appliquée, 15, 9-20.

DE COCK, Sylvie. (2004). Preferred Sequences of Words in NS and NNS Speech. Belgian Journal of English Language and Literatures (BELL), New Series 2, 225-246.

FALAISE, Achille, Tutin, Agnès \& KRAIF, Olivier. (2011). Une interface pour l'exploitation de corpus arborés par des non-informaticiens : la plate-forme ScienQuest du projet Scientext. Traitement automatique des langues, 52(3), 103-128.

FALAISE, Achille. (2013). Adaptation de la plateforme corporale ScienQuest pour l'aide à la rédaction en langue seconde. Dans Actes de TALN 2013, Les Sables-d'Olonne.

FRÉROT, Cécile, Rossi, Caroline \& FALAISE, Achille. (2014). Integrating Selected Corpus Data in the Classroom: A Case-Study of English NPs for French Students in Specialized Translation. Dans Proceedings of the 6th International Conference on Corpus Linguistics, Las Palmas de Gran Canaria, Espagne.

GRANGER, Sylviane \& PAQUOT, Magali. (2010). Customising a General EAP Dictionary to Meet Learner Needs. Dans S. Granger \& M. Paquot (dir.), eLexicography in the 21st Century: New Challenges, New Applications (p. 87-96). Louvain-la-Neuve : Presses universitaires de Louvain.

HARTWELL, Laura \& JACQUES, Marie-Paule. (2012). A Corpus-Informed Text Reconstruction Resource for Learning about the Language of Scientific Abstracts. Communication présentée à la conférence « EUROCALL 2012 », Göteborg, Suède. 
JACQUeS, Marie-Paule, HARTWELl, Laura \& FALAISE, Achille. (2013). TAL et linguistique de corpus pour aider la rédaction scientifique en anglais. Dans Actes de TALN 2013 (p. 12-26), Les Sablesd'olonne.

JoHns, Tim. (1991). Should You Be Persuaded: Two Examples of Data-Driven Learning. English Language Research Journal, 4, 1-16.

LANDURE, Corinne. (2013). Corpus et dictionnaires : prise de conscience linguistique chez des apprenants LANSAD. Bulletin VALS-ASLA, 97, 59-76.

MANGIANTE, Jean-Marc \& CHANTAL, Parpette. (2011). Le français sur objectif universitaire. Grenoble : Presses universitaires de Grenoble.

Mauranen, Anna. (2004). Speech Corpora in the Classroom. Dans G. Aston, S. Bernardini \& D. Stewart (dir.), Corpora and Language Learners (p. 195-211). Amsterdam : John Benjamins.

OSBORNE, John. (1994). La cohésion dans les productions écrites d'étudiants en anglais de spécialité : un problème culturel ? ASp (la revue du GERAS), 5-6, 205-216.

PAQUOT, Magali. (2010). Academic Vocabulary in Learner Writing: From Extraction to Analysis. Londres-New York : Continuum International Publishing Group.

SIMARD, Claude. (1994). Pour un enseignement plus systématique du lexique. Québec français, 92, 28-33.

TRAN, Thi Thu Hoai (2014). Développement d'une aide à l'écrit scientifique. Description de la phraséologie scientifique et réflexion didactique pour l'enseignement à des étudiants non natifs (Thèse de doctorat en sciences du langage - spécialité français langue étrangère). Université Grenoble Alpes, Grenoble.

Tutin, Agnès. (2010). Sens et combinatoire lexicale : de la langue au discours (Thèse d'habilitation à diriger des recherches). Université Stendhal-Grenoble 3, Grenoble.

Tutin, Agnès (2007). Autour du lexique et de la phraséologie des écrits scientifiques. Revue française de linguistique appliquée, 12(2), 5-14.

Tutin, Agnès \& GrossmanN, Francis (dir.). (2012). Autour du corpus Scientext : de la constitution d'un corpus d'écrits scientifiques à l'étude des marques du positionnement et du raisonnement. Rennes : Presses universitaires de Rennes.

Tutin, Agnès \& FALAISE, Achille. (2013). Multiword Expressions in Scientific Discourse: A CorpusDriven Database. Dans Actes de eLex 2013, Tallinn, Estonie.

WRAY, Alison (2002). Formulaic Sequences and the Lexicon. Cambridge : Cambridge University Press.

\section{ANNEXES}

\section{ANNEXE 1}

\section{Questionnaire 1. - Évaluation d'un dictionnaire d'aide à la rédaction universitaire}

Pour répondre aux questions dans cette partie, veuillez consulter l'adresse suivante : http://dicorpus.aiakide.net/ht/?b=base_marqueurs_dicorpus.

Il s'agit d'un dictionnaire créé par notre équipe pour vous accompagner dans la rédaction de rapport de stage ou de mémoire. Pour chaque connecteur logique, vous avez des informations concernant sa catégorie syntaxique, sa fréquence, etc. 
1.1. Comment trouvez-vous l'interface de ce dictionnaire?

Sympa, convivial

Trop simple

Quelles sont vos suggestions d'amélioration?

Pour chercher des informations pour le mot « d'abord », suivez le chemin suivant : Mode d'accès : Accès par sens > Pour exprimer : une énumération > Expression : d'abord.

Observez le tableau de la catégorie, la position dans la phrase, etc. et les exemples en bas de la page. Il s'agit des exemples extraits des articles scientifiques. Cliquez sur « d'abord» dans le tableau des exemples, vous pouvez visualiser un contexte plus large.

Vous pouvez renouveler les mêmes recherches pour d'autres connecteurs logiques.

1.2. Est-ce que vous trouvez les informations nécessaires en consultant ce dictionnaire?

Oui

Oui, mais pas tout

Non

(Veuillez préciser) car vous cherchez essentiellement

1.3. Cliquez à Mode d'accès : Accès par sens > Pour exprimer : enchainement thématique > Sous catégorie : insister sur une idée > Expression : à ce propos

Est-ce que ces notions sont faciles à comprendre ? (enchainement thématique, insister sur une idée, etc.) (Plusieurs réponses sont possibles)

Oui, je les comprends

Non, je ne les comprends pas, car

$\square$ Les termes sont compliqués.

$\square$ Je ne comprends pas ce que signifie « insister sur une idée ».

1.4. Est-ce que vous comprenez la différence entre les deux modes d'accès « Accès par sens » et « Accès par expression »?

Oui

Oui, un peu, je comprends que

Non, je ne comprends pas très bien. À mon avis, ça signifie :

1.5. Sur la forme, comment trouvez-vous les exemples proposés en bas de page?

Un peu difficile de les trouver, car il faut descendre la page.

Pas difficile.

1.6. Sur le fond, est-ce que vous avez trouvé les exemples que vous souhaitez ?

Oui, il y a beaucoup d'exemples, c'est très bien.

Non, il y a trop d'exemples, je ne sais pas quel exemple choisir.

1.7. Quelle est votre impression générale en consultant ce dictionnaire ? (Plusieurs réponses sont possibles)

C'est très bien d'avoir ce genre de dictionnaire.

Je souhaite que ce soit plus convivial.

Je souhaite que les exemples soient filtrés par discipline, je veux consulter uniquement les exemples dans ma discipline de spécialité.

Votre propre suggestion : 
ANNEXE 2

\section{Questionnaire 2. - Évaluation d'un dictionnaire d'aide à la rédaction universitaire}

1. Quels sont les dictionnaires que vous utilisez quand vous rédigez?

2. Comment trouvez-vous l'interface de ce dictionnaire? (sympa, convivial, trop simple, etc.)

Quelles sont vos suggestions d'amélioration?

3. Quelles sont les informations que vous avez trouvées en consultant ce dictionnaire?

4. Quelles sont les notions les plus compliquées pour vous dans ce dictionnaire?

5. Quelle est votre impression générale en consultant ce dictionnaire ? (difficile à consulter, trop d'exemples, utile mais manque de dynamisme, etc.)

Votre propre suggestion :

6. Quels sont vos objectifs en vous inscrivant à ce DU ? Quelles sont vos appréciations en suivant cette formation?

\section{NOTES}

1. <https://dicorpus.aiakide.net>.

2. ARTES (Aide à la rédaction des textes scientifiques) : <https://artes.eila.univ-paris-diderot.fr/

$>$.

3. Projet ANR Scientext piloté par Agnès Tutin et Francis Grossmann (2006-2010), adresse du projet Scientext : <http://scientext.msh-alpes.fr/scientext-site/spip.php?article1>.

4. En utilisant le corpus de textes scientifiques anglais du méta-corpus Scientext.

5. Diplôme universitaire français langue étrangère pour la préparation aux études supérieures.

6. Les phrases entre guillemets sont les appréciations des étudiants, certaines formulations ont été corrigées grammaticalement.

\section{RÉSUMÉS}

Nous présentons dans cet article Dicorpus, un prototype permettant d'associer une base lexicale et un corpus en français, les expérimentations que nous avons menées sur cet outil auprès d'étudiants apprenant le français, et les modifications effectuées sur cet outil en réponse aux besoins mis en évidence au cours des expérimentations.

This paper introduces Dicorpus, a prototype allowing the association of a French lexical base and corpus, the experiments that we conducted on this tool with students learning French, and the modifications made on this tool in response to the needs highlighted during the experiments. 
INDEX

Mots-clés : Dicorpus, rédaction scientifique, FLE

Keywords : Dicorpus, academic writing, FFL

\section{AUTEURS}

THI THU HOAI TRAN

GRAMMATICA, EA 4521, Université d'Artois

\section{ACHILLE FALAISE}

ICAR, UMR 5191, CNRS / LLF, UMR 7110, CNRS 